\title{
Safety of human papillomavirus vaccines in healthy young women: a meta-analysis of 24 controlled studies
}

\author{
Yukari Ogawa ${ }^{*}$, Hinako Takei ${ }^{1}$, Ryuichi Ogawa ${ }^{2}$ and Kiyoshi Mihara ${ }^{1}$
}

\begin{abstract}
Background: Human papillomavirus (HPV) vaccines have been shown to be effective for the eradication of HPV and prevention of cervical cancer. However, the number of women who receive HPV vaccinations has decreased over the last several years in Japan, due to concerns about adverse reactions associated with the vaccines. We evaluated the safety of three types of HPV vaccines separately in young women and the difference in the risk of adverse reactions between HPV and other vaccines by conducting a meta-analysis.
\end{abstract}

Methods: Primary literature was retrieved from MEDLINE, the Cochrane Central Register of Controlled Trials, and Japana Centra Revuo Medicina. Prospective controlled studies with participants consisting exclusively of healthy women who received bivalent, quadrivalent, or 9-valent HPV (2vHPV, 4VHPV or 9vHPV) vaccines were included. Primary safety outcome was the incidence of solicited local and systemic symptoms, and unsolicited symptoms. When two or more studies were found for the same analysis, a meta-analysis was applied.

Results: A total of 24 controlled studies from 22 articles were included in our study. Of the 24 studies, 16 were placebo-controlled and eight were active-controlled (different HPV vaccine or hepatitis vaccine). Average ages of the participants ranged from 12 to 37 years. A significantly higher incidence of solicited local symptoms was observed following injection of HPV vaccines (2vHPV and 4VHPV) compared to placebo, but there was no difference between HPV vaccines [risk ratio (RR) for 2vHPV: 1.25, 95\% confidence interval (Cl): 1.09 to 1.43, RR for 4VHPV: 1.16, 95\% Cl: 1.11 to 1.20]. The incidence of solicited systemic symptoms was not different between HPV vaccines and placebo (RR: 1.04, 95\% Cl: 0.99 to 1.09). The incidence of unsolicited symptoms was significantly higher for 2vHPV vaccine compared to placebo (RR: $1.28,95 \% \mathrm{Cl}: 1.01$ to 1.63 ), but was not significantly different between 2vHPV and hepatitis B vaccines.

Conclusions: HPV vaccines had significantly higher risk of any injection site symptom compared to placebo or other vaccines (hepatitis A and B vaccines), and the incidence of solicited local symptoms was no difference between 2VHPV vaccination and 4VHPV vaccination. However, the most adverse reactions were transient.

Keywords: HPV vaccine, Cervical cancer, Solicited local symptoms, Solicited systemic symptoms, Safety, Meta-analysis

\footnotetext{
* Correspondence: y_ogawa@musashino-u.ac.jp

${ }^{1}$ Faculty of Pharmacy, Department of Pharmaceutical Sciences, Musashino

University, 1-1-20 Shinmachi Nishitokyo-shi, Tokyo 202-8585, Japan

Full list of author information is available at the end of the article
} 


\section{Background}

Cervical cancer is the fourth most common cancer among women worldwide [1, 2] and a major world health problem for women. The estimated number of cervical cancer cases in 2012 was 528,000, and the number of deaths was 266,000 [3] in the world. In Japan, 2813 persons died from the disease in 2015, with crude mortality rate of 4.4 per 100 thousand population [4]. Both incidence and mortality of cervical cancer in young women have been reported to increase over the last 20 years [4]. Furthermore, it is forecasted that 10,600 to 12,600 cases of cervical cancer will be diagnosed annually in 2015 through 2029 in Japan, and 2600 to 2800 will die from the disease [5]. Persistent HPV infection is the most important factor for the development of cervical cancer [6, 7]. Although most of the patients who are infected by human papillomavirus (HPV) present no symptoms, long-standing HPV infection may lead to development of cervical cancer in women.

Of the 15 oncogenic HPV types identified, HPV-16 and HPV-18 account for nearly 70\% of all invasive cervical cancer cases worldwide, with HPV-45, HPV-33 and HPV-31 accounting for approximately $10 \%$ of reported cases [8]. HPV-16 and HPV-18 also account for 50.3\% of all invasive cervical cancer cases in Japan [9]. Since HPV infections are transmitted through sexual contact, girls aged 9 to 14 years are the primary target of HPV vaccination in most of the countries. A clinical trial has shown that prophylactic HPV vaccination is highly efficacious for the prevention of HPV infection and the associated cervical intraepithelial neoplasia [10]. Among three internationally marketed HPV vaccines [bivalent HPV (2vHPV), quadrivalent HPV (4vHPV), and 9-valent HPV (9vHPV) vaccines], 2vHPV and 4vHPV are now being marketed in Japan.

In 2009, the World Health Organization (WHO) issued the first position paper on the inclusion of routine $\mathrm{HPV}$ vaccination in national immunization programs [11]. In Japan, financial support from the government was initiated in 2011, and the Ministry of Health, Labour, and Welfare (MHLW) started to promote HPV vaccination in April 2013. However, in May and June 2013, Japanese news media distributed a concern regarding the potential of the vaccine to cause undesirable reactions such as chronic pain, together with sensational coverage of other negative information. In June 2013, MHLW withdrew the recommendation of HPV vaccination [12]. The news of adverse events associated with $\mathrm{HPV}$ vaccination strongly affected the vaccination rate in Japan [13], with a substantial decrease in 2013 compared to 2012 [14].

A few meta-analyses have reported the safety of HPV vaccines regarding solicited local and systemic symptoms to date $[15,16]$. However, none of the previous meta-analyses evaluated the safety in relation to types of $\mathrm{HPV}$ vaccine (i.e., 2vHPV, $4 \mathrm{vHPV}$, and 9vHPV), and these studies compared the safety with combined controls (i.e., placebo and active controls). Therefore, we performed a meta-analysis to re-evaluate the safety of three types of HPV vaccines separately and to evaluate whether the frequency of adverse reactions after HPV vaccination is higher than that after other vaccinations such as hepatitis vaccines in healthy young women, using controlled studies published to date and unpublished studies including Japanese studies.

\section{Methods \\ Data sources and searches}

We searched MEDLINE (1966 to February, 2017), the Cochrane Central Register of Controlled Trials (issue 7, 2016), and Japana Centra Revuo Medicina (1981 to February, 2017). We combined the MeSH terms or text words of "papillomavirus vaccine" and "adverse reactions". We included original research articles written in either English or Japanese. In addition, we manually searched the reference lists of all the selected studies and related articles. Additionally, we reviewed the Pharmaceuticals and Medicines Devices Agency (PMDA) website [17] for any additional unpublished studies relevant to the topic.

\section{Study selection}

The inclusion criteria of the present study were: (1) prospective controlled studies that evaluated safety (solicited local symptoms, solicited systemic symptoms, and unsolicited symptoms); (2) studies that included only healthy women aged nine years or above; and (3) studies in which participants were administered 2vHPV, $4 \mathrm{vHPV}$, or 9vHPV vaccine. Clinical studies in which more than two vaccinations were performed concomitantly and in which all participants were older than 40 years were excluded. Two investigators ( $\mathrm{YO}$ and HT) applied the inclusion and exclusion criteria independently. When there was a disagreement between the two investigators, a final decision was made after careful discussion.

\section{Data extraction}

Study design (random allocation, blinded); number of subjects; type of HPV vaccine and control used; country or region in which the study was performed; and incidence of solicited local symptoms, solicited systemic symptoms and unsolicited symptoms were extracted from each study. We defined these symptoms as follows [18-20]: Solicited local symptoms were defined as pain, redness and/or swelling at the injection site observed within 15 days after vaccination. Solicited systemic symptoms were defined as arthralgia, fatigue, fever, 
gastrointestinal symptoms, headache, rash, urticaria, and/or myalgia observed within 15 days after vaccination. All other undesirable reactions occurring within 30 days after injection of the vaccine were included as unsolicited symptoms. We also extracted the incidence of grade 3 solicited local symptoms defined as pain that prevents normal activity, and redness or swelling at the injection site with a diameter greater than $50 \mathrm{~mm}$ [21-23].

Primary outcome in the present meta-analysis was the overall incidence of solicited local and systemic symptoms, and that of unsolicited symptoms after HPV vaccination. Secondary outcomes were all the components of the primary outcome as well as cumulative incidence of grade 3 solicited local symptoms, solicited systemic symptoms, and unsolicited symptoms.

\section{Assessment of risk of bias in included studies}

The quality of each included study was assessed using Cochrane's risk of bias tool [24]. The tool consists of seven categories: random sequence generation (selection bias), allocation concealment (selection bias), blinding of participants and personnel (performance bias), blinding of outcome assessment (detection bias), incomplete outcome data (attention bias), selective reporting (reporting bias) and other bias. We assessed the risk of bias as low, high, or unclear, and the quality of each study is presented in a "risk of bias" table.

\section{Data synthesis and analysis}

We used risk ratio (RR) as a measure of effect for outcomes. We conducted meta-analysis when data of at least two studies were available, and calculated pooled RRs with 95\% confidence intervals (CI) using randomeffects methods (Mantel-Haenszel). Inter-study heterogeneity was assessed by $\mathrm{I}^{2}$ statistic $\left(\mathrm{I}^{2}>75 \%\right.$ indicates substantial heterogeneity) [25]. Data were analyzed by Cochrane Review Manager version 5.3 (The Nordic Cochrane Centre, The Cochrane Collaboration).

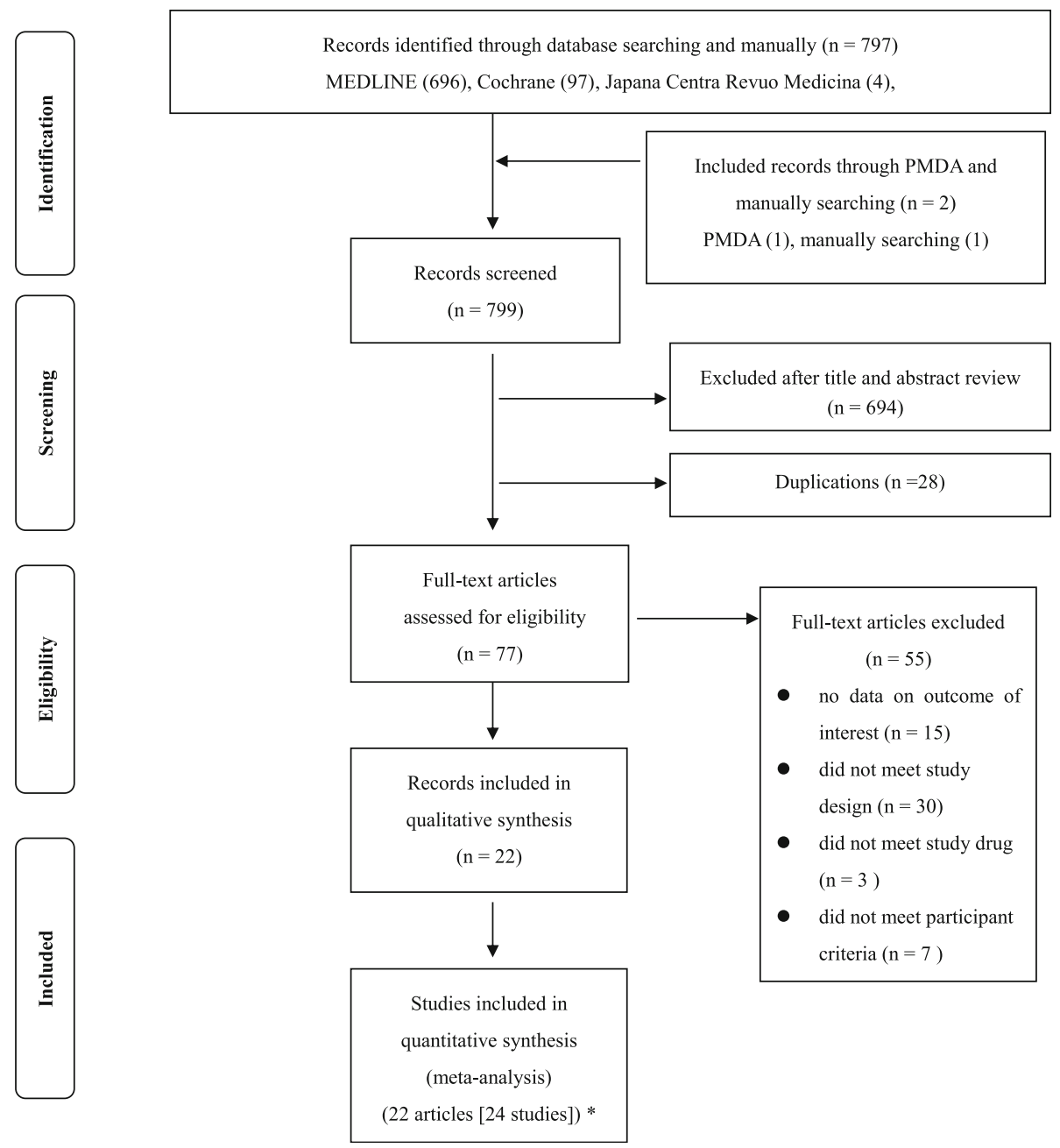

Fig. 1 A study selection flow diagram. n: total number of articles. *We included 24 studied from 22 articles because 2articles [26, 47] included 2 studies each. Abbreviations: PMDA, Pharmaceutical Medicines Devices Agency 


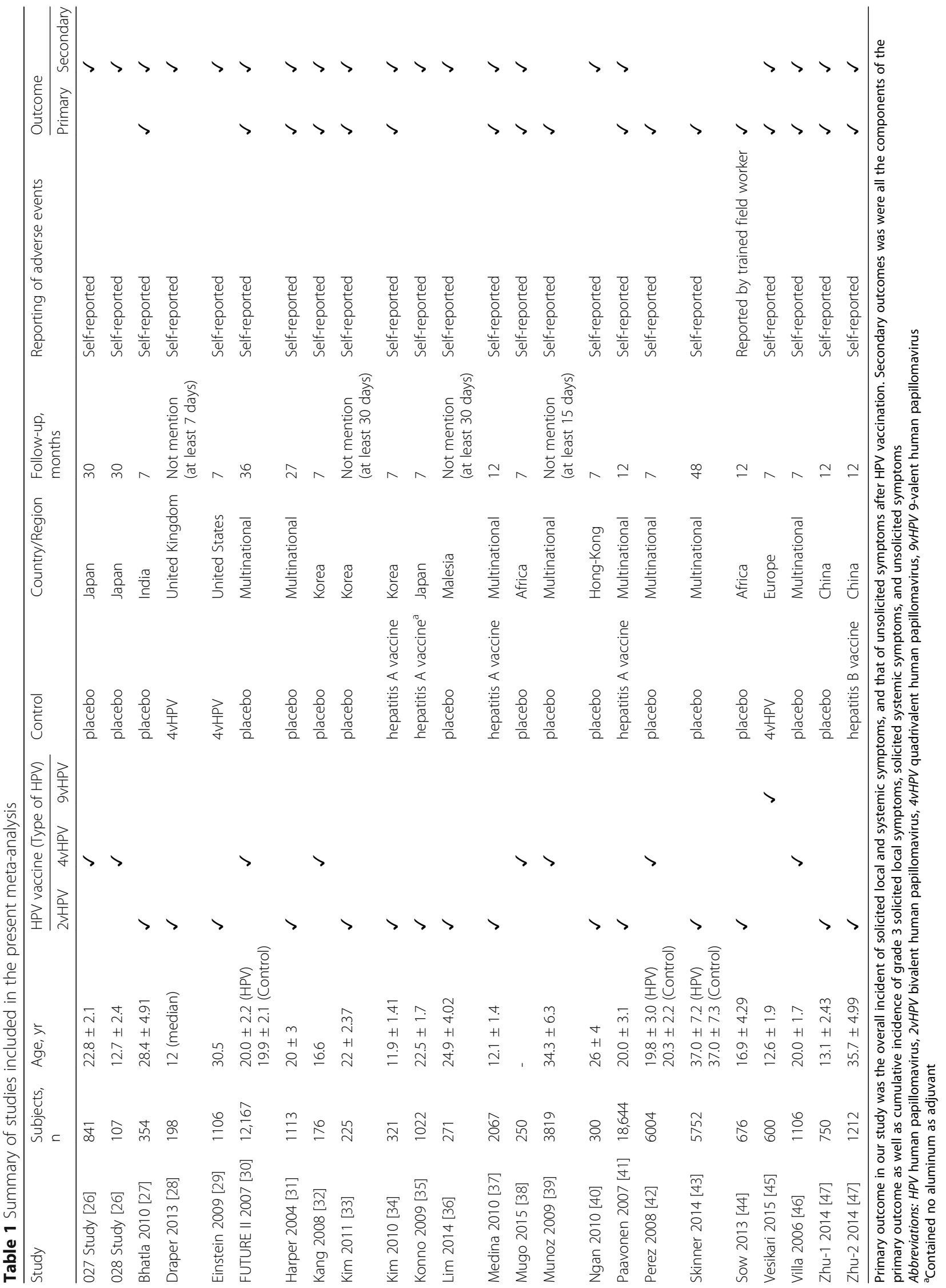




\section{Results}

\section{Study retrieval and characteristics of included studies}

The primary literature search retrieved 799 articles as follows: MEDLINE (696), Cochrane (97), Japana Centra Revuo Medicina (4), PMDA website (1), manually searching (1). (Fig. 1). Of the 799 articles, 777 were excluded. The reasons for exclusion were: title and abstract review indicated no relevance to our study objective (694), duplications (28), no data on outcome of interest (15), uncontrolled design (30), experimental vaccination (3), and male study or patients complicated with infectious diseases (7). The remaining 22 original research papers [26-47] were included in our analysis. Since two of the papers $[26,47]$ contained two studies each, we analyzed a total of 24 studies. The number of participants, mean age, HPV vaccine tested, and control of each included study are summarized in Table 1 . All the studies were randomized controlled trials. Average ages of participants ranged from 12 to 37 years. Geographic background in the 24 included studies was as follows: Japan (3), other Asian countries (8), Europe (2), Africa (2), United States, and multinational (9). Three studies [28, $29,45]$ used HPV vaccines as active controls. The symptoms consisted of the defined adverse reactions after the vaccinations were self-reported in all included studies except for one report [44].

Results of risk of bias assessments of the included studies are shown in Fig. 2. Analysis using the Cochrane risk of bias tool indicated an overall low risk of bias. One study [42] had insufficient information on discontinued data. The risks of bias due to inadequate random sequence generation and inadequate allocation concealment were judged to be 'unclear' in seven studies [26, 29-31, 35, 38] and four studies $[26,33,38]$, respectively.

\section{Safety of HPV vaccines}

\section{Solicited local symptoms}

We analyzed nine placebo-controlled studies (four $2 \mathrm{vHPV}$ vaccine $[27,31,43,44]$ and five $4 \mathrm{vHPV}$ vaccine studies $[30,38,39,42,46])$ for the primary safety outcome. A meta-analysis showed a significantly higher incidence of any solicited local symptom in the pooled HPV vaccine (2vHPV and 4vHPV) group compared to the placebo group (overall RR: 1.20, 95\% CI: 1.13 to 1.27 ) (Fig. 3). There was no difference between types of HPV vaccine (pooled RR for 2vHPV: 1.25 [1.09 to 1.43], pooled RR for 4vHPV 1.16 [1.11 to 1.20$]$ ).

All HPV vaccines were associated with significantly increased incidence in all components of solicited local symptoms (pain, redness and swelling at the injection sites) (Table 2). In five hepatitis vaccinecontrolled studies, the incidence of pain, redness,

\begin{tabular}{|c|c|c|c|c|c|c|c|}
\hline & 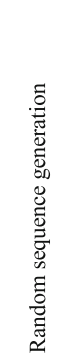 & 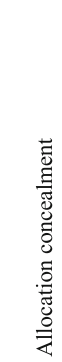 & 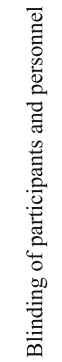 & 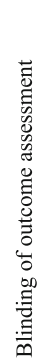 & $\begin{array}{l}\text { J } \\
\text { J } \\
\text { : } \\
0 \\
0 \\
0 \\
\frac{0}{2} \\
\text { : } \\
0 \\
0\end{array}$ & 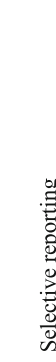 & 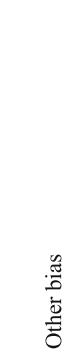 \\
\hline 027 Study & ? & ? & ? & ? & $\oplus$ & $\oplus$ & $\oplus$ \\
\hline 028 Study & $?$ & ? & ? & ? & $\oplus$ & $\oplus$ & $\oplus$ \\
\hline Bhatla 2010 & 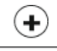 & $\oplus$ & † & $\mp$ & $\oplus$ & 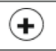 & ( \\
\hline Draper 2013 & $\oplus$ & $\oplus$ & 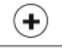 & † & $\oplus$ & $\uplus$ & ? \\
\hline Einstein 2009 & ? & $\oplus$ & $\oplus$ & ? & ? & ? & ? \\
\hline FUTURE II 2007 & $?$ & $\oplus$ & $\oplus$ & ? & 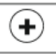 & $\oplus$ & ? \\
\hline Harper 2004 & ? & $\oplus$ & $\oplus$ & $\oplus$ & $\oplus$ & $\oplus$ & ? \\
\hline Kang 2008 & 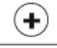 & $\oplus$ & $\oplus$ & ? & $\oplus$ & ? & ? \\
\hline Kim 2010 & 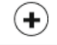 & $\oplus$ & 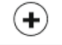 & ? & $\oplus$ & $\oplus$ & ? \\
\hline Kim 2011 & 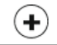 & ? & ? & $\oplus$ & $\oplus$ & ? & ? \\
\hline Konno 2009 & ? & $\oplus$ & ? & $\oplus$ & 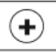 & $\oplus$ & ? \\
\hline $\operatorname{Lim} 2014$ & $\oplus$ & $\oplus$ & 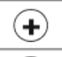 & ? & $\oplus$ & ? & ? \\
\hline Medina 2010 & 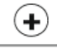 & $\oplus$ & † & ? & † & $\oplus$ & ? \\
\hline Mugo 2015 & ? & ? & $\oplus$ & ? & 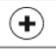 & $\oplus$ & ? \\
\hline Munoz 2009 & $\mp$ & 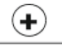 & 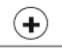 & ? & $\oplus$ & $\oplus$ & ? \\
\hline Ngan 2010 & 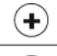 & $\oplus$ & 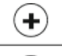 & ? & † & ? & ? \\
\hline Paavonen 2007 & 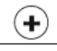 & 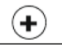 & 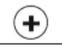 & ? & 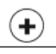 & $\oplus$ & ? \\
\hline Perez 2008 & $\oplus$ & $\oplus$ & $\oplus$ & ? & - & $\oplus$ & ? \\
\hline Skinner 2014 & 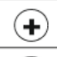 & $\oplus$ & ( & 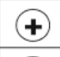 & 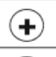 & $\oplus$ & ? \\
\hline Sow 2013 & $\oplus$ & 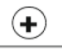 & 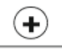 & $\oplus$ & $\oplus$ & ? & ? \\
\hline Vesikari 2015 & 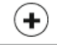 & $\oplus$ & 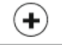 & ? & $\oplus$ & $\oplus$ & ? \\
\hline Villa 2006 & 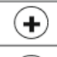 & $\oplus$ & $\uplus$ & $?$ & 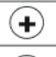 & ? & ? \\
\hline Zhu-1 2014 & 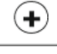 & $\oplus$ & † & 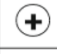 & 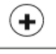 & ? & ? \\
\hline Zhu-2 2014 & † & $\oplus$ & 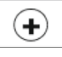 & $\mp$ & $\oplus$ & ? & ? \\
\hline \multicolumn{8}{|c|}{$\begin{array}{l}\text { Fig. } 2 \text { Summary of risk of bias for the } 24 \text { studies included in } \\
\text { meta-analysis. The judgements were made by two of the study } \\
\text { investigators. Symbols: +, low risk of bias; -, high risk of bias;?, } \\
\text { bias risk is unclear }\end{array}$} \\
\hline
\end{tabular}

and swelling was significantly higher in the 2vHPV vaccine group than in the hepatitis vaccine group (pooled RRs [95\% CIs] for pain: 1.80 [1.29 to 2.51], redness 1.77 [1.55 to 2.02], and swelling 2.57 [2.19 to 3.02]). Our study found no hepatitis vaccine-controlled study of $4 \mathrm{vHPV}$ vaccine. Two studies mentioned that there were subjects discontinued due to serious injection-site symptom. In one study, one subject (less than $0.1 \%$ ) in the placebo group discontinued due to hypersensitivity. In another study, five subjects $(0.3 \%)$ in 4vHPV group withdrew due to solicited local 


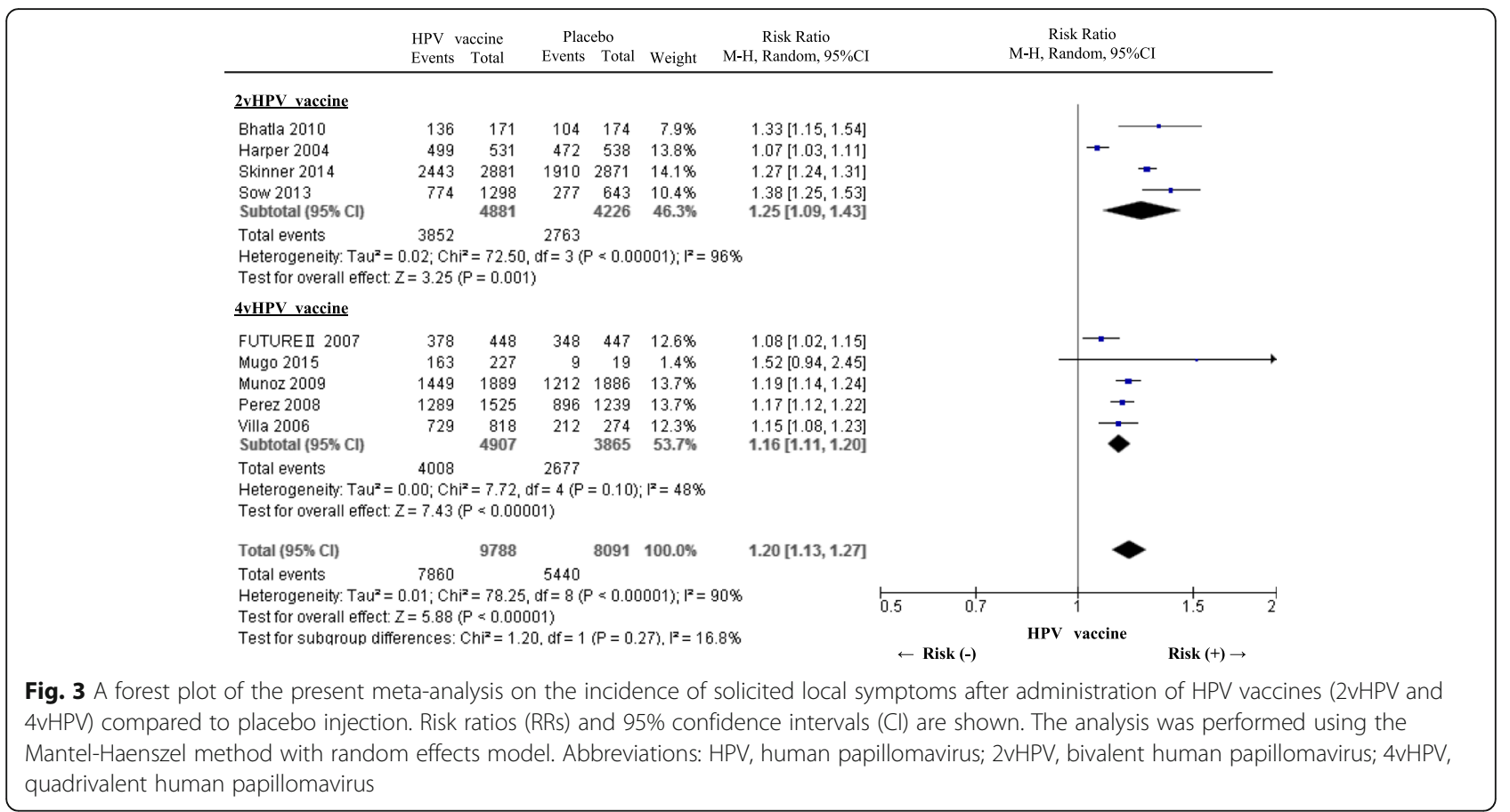

symptoms. In others, adverse reactions were transient, and there was no participant who discontinued the study schedule owing to the solicited local symptoms.

2. Grade 3 solicited local symptoms

We gathered information on grade 3 solicited local symptoms from the $2 \mathrm{vHPV}$ vaccine studies (three hepatitis vaccine-controlled studies [34, 37, 41] and two placebo-controlled studies [27, 40]) (Table 3). The $2 \mathrm{vHPV}$ vaccine showed a significantly higher incidence of pain compared to placebo, with RR [95\% CI] of 6.58 [3.03 to 4.30]. In addition, the $2 \mathrm{vHPV}$ vaccine was associated with higher incidence of grade 3 symptoms compared to hepatitis vaccine, with pooled RRs [95\% CIs] of 4.49 [3.02 to 6.68] for pain, 6.30 [1.97 to 20.16] for redness, and 5.04 [3.21 to 7.91] for swelling. The durations of the symptoms, however, were similar in 2vHPV and control groups. Three of the five studies $[34,40,41]$ reported that the symptoms lasted no longer than 4-5 days in both groups, and the remaining two studies did not report the duration of symptoms [27, 37]. Only one study tested 4vHPV [38] reported grade 3 pain, and there was no significant difference in the pain compared with the placebo arm (RR: 0.50 [0.06 to 3.96]).

Table 2 Pooled risk ratios of solicited local symptoms

\begin{tabular}{|c|c|c|c|c|}
\hline Comparison & Studies, n & Subjects, n & Pooled RR [95\% Cl] & Reference \\
\hline \multicolumn{5}{|l|}{ Pain } \\
\hline 2 vHPV vaccine vs Hepatitis vaccine ${ }^{a}$ & 5 & 15,471 & $1.80[1.29,2.51]$ & {$[34,35,37,41,47]$} \\
\hline 2vHPVvaccine vs Placebo & 7 & 5315 & $1.34[1.15,1.57]$ & {$[27,31,33,36,40,44,47]$} \\
\hline 4vHPVvaccine vs Placebo & 6 & 3474 & $1.17[1.10,1.24]$ & {$[26,30,32,38,46]$} \\
\hline \multicolumn{5}{|l|}{ Redness } \\
\hline 2 VHPV vaccine vs Hepatitis vaccine ${ }^{a}$ & 5 & 15,471 & $1.77[1.55,2.02]$ & {$[34,35,37,41,47]$} \\
\hline 2vHPV vaccine vs Placebo & 6 & 3374 & $1.77[1.52,2.07]$ & {$[27,31,33,36,40,47]$} \\
\hline $4 \mathrm{VHPV}$ vaccine vs Placebo & 2 & 1055 & $1.47[1.18,1.82]$ & [26] \\
\hline \multicolumn{5}{|l|}{ Swelling } \\
\hline 2vHPV vaccine vs Hepatitis vaccine ${ }^{a}$ & 5 & 15,471 & $2.57[2.19,3.02]$ & {$[34,35,37,41,47]$} \\
\hline 2vHPV vaccine vs Placebo & 7 & 5315 & $2.27[1.78,2.91]$ & {$[27,31,33,36,40,44,47]$} \\
\hline 4vHPV vaccine vs Placebo & 4 & 2403 & $1.83[1.50,2.22]$ & {$[26,38,46]$} \\
\hline
\end{tabular}

Abbreviations: HPV human papillomavirus, $2 \mathrm{VHPV}$ bivalent human papillomavirus, $4 \mathrm{vHPV}$ quadrivalent human papillomavirus, $R R$ risk ratio, $\mathrm{Cl}$ confidence interval ${ }^{a}$ Four studies $[34,35,37,41]$ used hepatitis $A$ and one study [47] used hepatitis B vaccine as control 
Table 3 Pooled risk ratios of grade 3 solicited local symptoms

\begin{tabular}{|c|c|c|c|c|}
\hline Comparison & Studies, n & Subjects, $n$ & Pooled RR [95\% Cl] & Reference \\
\hline \multicolumn{5}{|l|}{ Pain } \\
\hline 2 VHPV vaccine vs Hepatitis vaccine ${ }^{a}$ & 3 & 13,237 & $4.49[3.02,6.68]$ & {$[34,37,41]$} \\
\hline 2vHPV vaccine vs Placebo & 2 & 645 & $6.58[3.03,4.30]$ & {$[27,40]$} \\
\hline \multicolumn{5}{|l|}{ Redness } \\
\hline 2 VHPV vaccine vs Hepatitis vaccine ${ }^{a}$ & 3 & 13,237 & $6.30[1.97,20.16]$ & {$[34,37,41]$} \\
\hline 2vHPV vaccine vs Placebo & 2 & 645 & $1.62[0.20,13.07]$ & {$[27,40]$} \\
\hline \multicolumn{5}{|l|}{ Swelling } \\
\hline 2vHPV vaccine vs Hepatitis vaccine ${ }^{a}$ & 3 & 13,237 & $5.04[3.21,7.91]$ & {$[34,37,41]$} \\
\hline 2vHPV vaccine vs Placebo & 2 & 645 & $2.52[0.58,10.97]$ & {$[27,40]$} \\
\hline
\end{tabular}

Grade 3 solicited local symptoms define as pain that prevents normal activity, and redness or swelling at the injection site with a diameter greater than $50 \mathrm{~mm}$ Abbreviations: HPV human papillomavirus, 2vHPV bivalent human papillomavirus, $R R$ risk ratio, $\mathrm{Cl}$ confidence interval

${ }^{a}$ All studies $[27,34,37,40,41]$ used hepatitis $A$ as control

\section{Solicited systemic symptoms}

We analyzed nine placebo-controlled studies (three $2 \mathrm{vHPV}$ vaccine $[31,43,44]$ and six $4 \mathrm{vHPV}$ vaccine studies [30, 32, 38, 39, 42, 46]). There was no significant difference when comparing $2 \mathrm{vHPV}$ or $4 \mathrm{vHPV}$ vaccine with placebo (Fig. 4). Comparing to placebo, 2vHPV vaccine group showed significantly higher RRs of fatigue, gastrointestinal symptoms, and myalgia but not in arthralgia, fever, rash, urticarial, and headache (Table 4). On the other hand, analysis of five hepatitis vaccine-controlled studies showed that the incidence of all components of solicited systemic symptoms except for gastrointestinal symptoms and myalgia was significantly higher in the $2 \mathrm{vHPV}$ vaccine group than in the hepatitis vaccine group (Table 4). Gastrointestinal symptoms and headache were components of interest in a meta-analysis of $4 \mathrm{vHPV}$ vaccine, and no significant differences were observed compared to placebo.

4. Unsolicited symptoms

Five placebo-controlled studies [27, 33, 43, 44, 47] and four hepatitis vaccine-controlled studies $[34,37,41,47]$ were available to assess the risk of unsolicited symptoms associated with 2vHPV vaccine. Three studies [34, 37, 41] used hepatitis A vaccine and one study [47] used hepatitis $B$ vaccine as control. The $2 \mathrm{vHPV}$ vaccine had a slightly but significantly higher risk of unsolicited symptoms compared to placebo (pooled RR [95\% CI]: 1.28 [1.01 to 1.63]), but there was no difference between $2 \mathrm{vHPV}$ vaccine and hepatitis vaccine (pooled RR [95\% CI]:

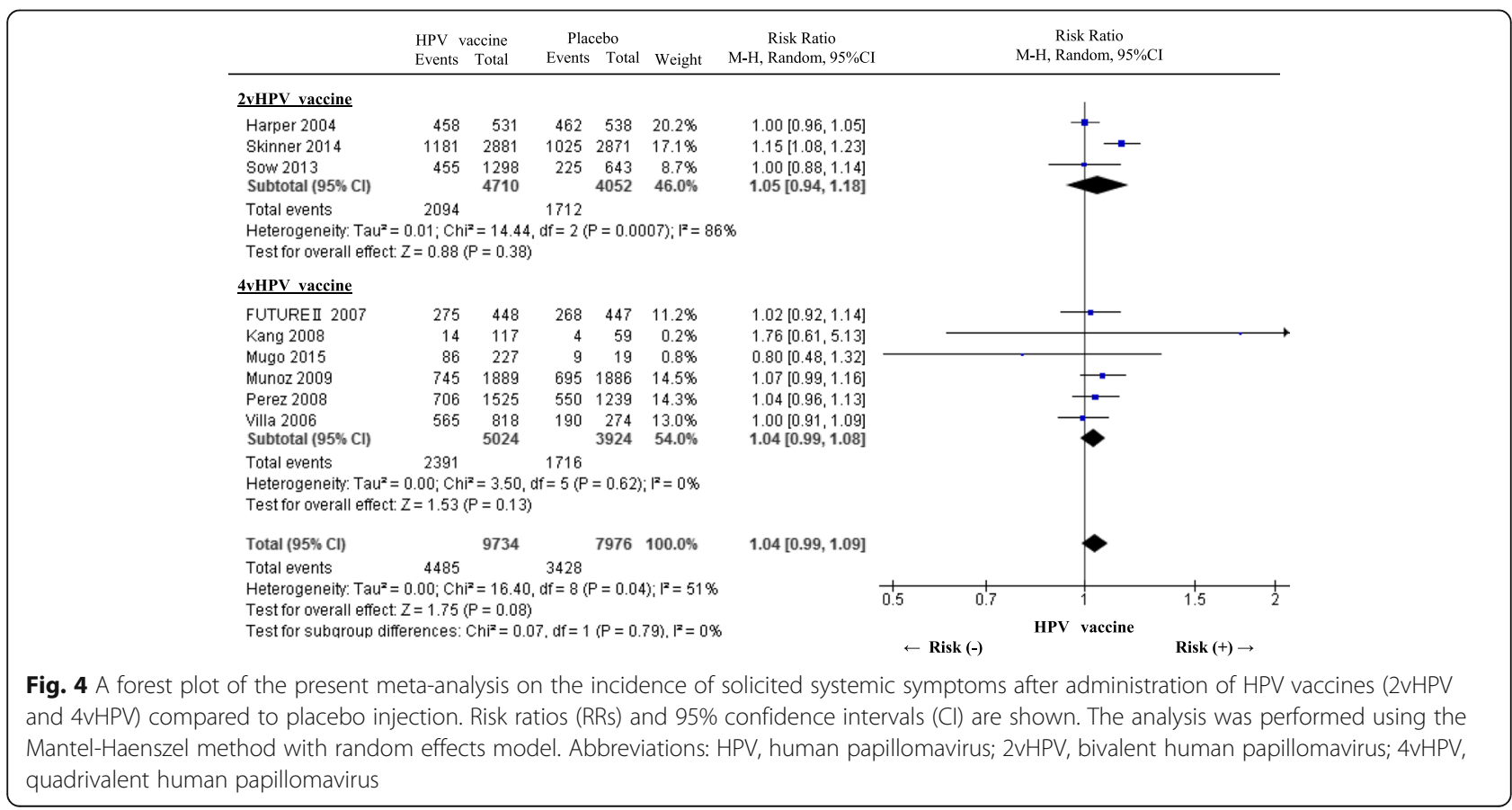


Table 4 Pooled risk ratios of solicited systemic symptoms

\begin{tabular}{|c|c|c|c|c|}
\hline Comparison & Studies, $n$ & Subjects, n & Pooled RR [95\% CI] & Reference \\
\hline \multicolumn{5}{|l|}{ Arthralgia } \\
\hline 2vHPV vaccine vs Hepatitis vaccine & 5 & 15,470 & $1.38[1.12,1.70]$ & {$[34,35,37,41,47]$} \\
\hline 2vHPVvaccine vs Placebo & 6 & 4246 & $1.03[0.40,2.69]$ & {$[27,33,36,40,44,47]$} \\
\hline \multicolumn{5}{|l|}{ Fatigue } \\
\hline 2vHPV vaccine vs Hepatitis vaccine $e^{a}$ & 5 & 15,470 & $1.13[1.05,1.22]$ & {$[34,35,37,41,47]$} \\
\hline 2vHPV vaccine vs Placebo & 7 & 5315 & $1.23[1.04,1.44]$ & {$[27,31,33,36,40,44,47]$} \\
\hline \multicolumn{5}{|l|}{ Fever } \\
\hline 2VHPV vaccine vs Hepatitis vaccine ${ }^{a}$ & 5 & 15,470 & $1.17[1.05,1.29]$ & {$[34,35,37,41,47]$} \\
\hline 2vHPV vaccine vs Placebo & 7 & 5315 & $1.11[0.95,1.28]$ & {$[27,31,33,36,40,44,47]$} \\
\hline \multicolumn{5}{|l|}{ Gastrointestinal symptoms } \\
\hline 2 vHPV vaccine vs Hepatitis vaccine ${ }^{a}$ & 5 & 15,470 & $1.03[0.97,1.10]$ & {$[34,35,37,41,47]$} \\
\hline 2vHPV vaccine vs Placebo & 7 & 10,796 & $1.46[1.06,2.02]$ & {$[27,31,33,40,43,44,47]$} \\
\hline $4 \mathrm{vHPV}$ vaccine vs Placebo & 2 & 1990 & $0.92[0.77,1.11]$ & \\
\hline \multicolumn{5}{|l|}{ Rash } \\
\hline 2vHPV vaccine vs Hepatitis vaccine ${ }^{a}$ & 5 & 15,470 & $1.43[1.15,1.77]$ & {$[34,35,37,41,47]$} \\
\hline 2vHPV vaccine vs Placebo & 7 & 10,796 & $1.26[0.80,1.99]$ & {$[27,31,33,40,43,44,47]$} \\
\hline \multicolumn{5}{|l|}{ Urticaria } \\
\hline 2 VHPV vaccine vs Hepatitis vaccine ${ }^{a}$ & 5 & 15,470 & $1.21[1.05,1.39]$ & {$[34,35,37,41,47]$} \\
\hline 2 vHPV vaccine vs Placebo & 6 & 9727 & $1.04[0.52,2.08]$ & {$[27,33,40,43,44,47]$} \\
\hline \multicolumn{5}{|l|}{ Headache } \\
\hline 2 vHPV vaccine vs Hepatitis vaccine ${ }^{a}$ & 5 & 15,470 & $1.09[1.01,1.18]$ & {$[34,35,37,41,47]$} \\
\hline 2 vHPV vaccine vs Placebo & 7 & 10,796 & $1.62[0.83,3.19]$ & {$[27,31,33,40,43,44,47]$} \\
\hline 4vHPV vaccine vs Placebo & 4 & 2396 & $1.01[0.85,1.19]$ & {$[26,38,46]$} \\
\hline \multicolumn{5}{|l|}{ Myalgia } \\
\hline 2vHPV vaccine vs Hepatitis vaccine ${ }^{a}$ & 5 & 15,470 & $1.07[0.721 .58]$ & {$[34,35,37,41,47]$} \\
\hline 2 vHPV vaccine vs Placebo & 6 & 9727 & $1.54[1.31,1.81]$ & {$[27,33,40,43,44,47]$} \\
\hline
\end{tabular}

Abbreviations: $H P V$ human papillomavirus, $2 v H P V$ bivalent human papillomavirus, $4 v H P V$ quadrivalent human papillomavirus, $R R$ risk ratio, $C l$ confidence interval ${ }^{a}$ Four studies $[34,35,37,41]$ used hepatitis $A$ and one study [47] used hepatitis B vaccine as control

1.02 [0.80 to 1.28$]$ ) (Fig. 5). The most frequently reported unsolicited symptoms were infections such as upper respiratory tract infection, nasopharyngitis, and dizziness. There was no data regarding $4 \mathrm{vHPV}$ vaccine.

5. $4 v H P V$ versus $2 v H P V$ or $9 v H P V$

We included three head-to-head comparison studies (4vHPV vaccine versus $2 \mathrm{vHPV}$ vaccine in two studies $[28,29]$, 4vHPV vaccine versus 9vHPV vaccine in one study [45]). Regarding solicited local symptoms, the $4 \mathrm{vHPV}$ vaccine had apparently lower risk of developing pain and redness than the other two HPV vaccines, but the differences did not reach statistical significance (pooled RR [95\% CI] for pain: 0.89 [0.75 to 1.05] and redness: 0.70 [0.46 to 1.07$]$ ). On the other hand, the $4 \mathrm{vHPV}$ vaccine was significantly lower risk of developing swelling (pooled RR [95\% CI]: 0.71 [0.57 to 0.88]). Regarding solicited systemic symptoms, 4vHPV vaccine had a significantly lower risk of fatigue compared to $2 \mathrm{vHPV}$ vaccine (pooled RR: 0.82, 95\% CI: 0.73 to 0.92 ) (Table 5 ).

\section{Discussion}

The $2 \mathrm{vHPV}$ vaccine and $4 \mathrm{vHPV}$ vaccine were licensed in 2009 and 2011, respectively, in Japan. The 9vHPV vaccine has been approved by the Food and Drug Administration in 2014 but not yet in Japan. We focused on the safety of $2 \mathrm{vHPV}$ and $4 \mathrm{vHPV}$ vaccines in the present study because MHLW has withdrawn the recommendation of HPV vaccination for adolescent women aiming to prevent cervical cancer, due to a concern over adverse reactions. Our meta-analysis summarized evidence-based safety profiles of HPV vaccines based on controlled studies including unpublished studies. The five major findings from our study are as follows. (1) Solicited local symptoms are more frequent after HPV vaccinations than after both placebo injection and hepatitis vaccination. (2) The risk of developing solicited local symptoms is no difference between $2 \mathrm{vHPV}$ vaccine and $4 \mathrm{vHPV}$ vaccine. (3) Most of observed solicited local symptoms such as pain, redness and swelling, even grade 3 symptoms, are transient. (4) The incidence of most of the solicited systemic symptoms is 

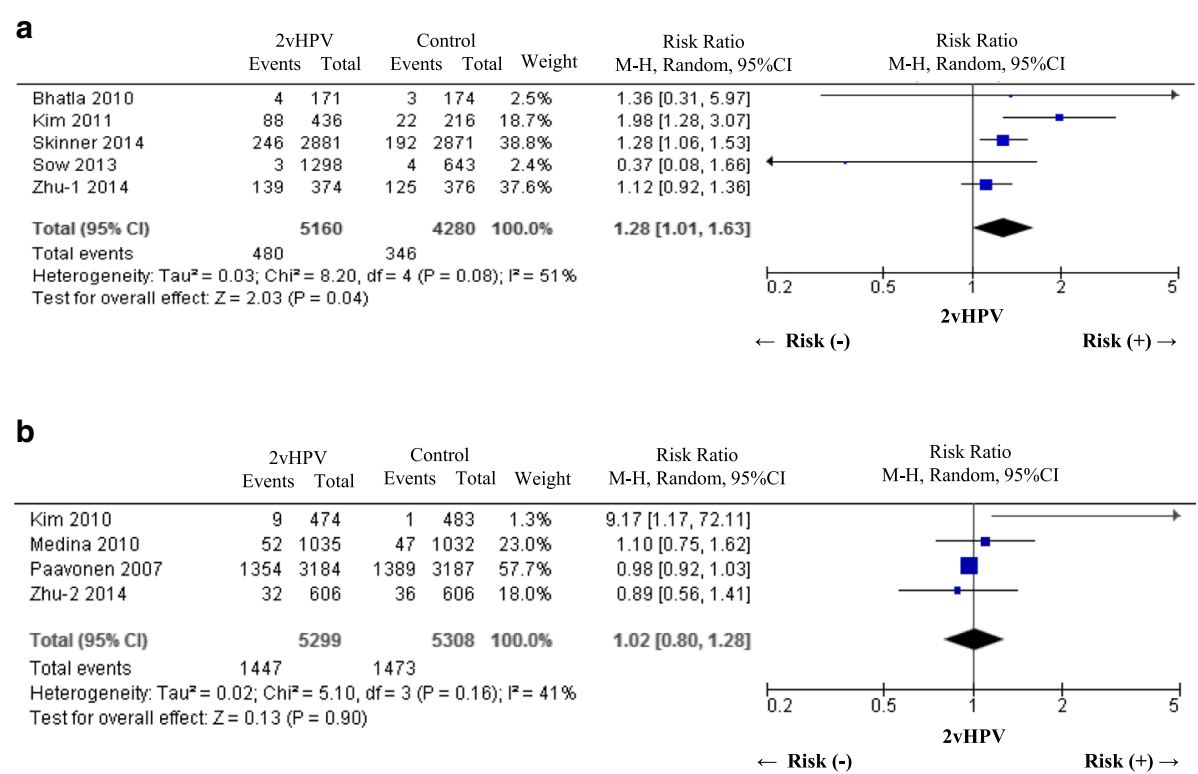

Fig. $5 \mathrm{~A}$ forest plot of the present meta-analysis on the incidence of unsolicited symptoms after administration of $2 \mathrm{vHPV}$ compared to placebo or hepatitis vaccine. a 2VHPV versus Placebo, b 2VHPV versus Hepatitis vaccine. Risk ratios (RRs) and 95\% confidence intervals (Cl) are shown. The analysis was performed using the Mantel-Haenszel method with random effects model. Abbreviations: HPV, human papillomavirus; 2vHPV, bivalent human papillomavirus

higher after HPV vaccination than after placebo injection or hepatitis vaccination. (5) Unsolicited symptoms after HPV vaccination appear to be more frequent compared to placebo injection, but similar to hepatitis vaccination.

There is a difference in pharmaceutical property between $2 \mathrm{vHPV}$ and $4 \mathrm{vHPV}$ vaccines. The $2 \mathrm{vHPV}$ vaccine contains the adjuvant system (AS)-04 composed of 3-O-desacyl4'-monophosphoryl lipid A (MPL) and aluminum salt (500 $\mu \mathrm{g}$ as aluminum). On the other hand, the $4 \mathrm{vHPV}$ vaccine contains only aluminum salt ( $225 \mu \mathrm{g}$ as aluminum). AS-04 is one of the new-generation adjuvants licensed for use in human vaccines, and has been developed to enhance vaccine-induced immune response $[48,49]$. MPL is derived from chemical modification of the potent immunomodulatory lipopolysaccharide (LPS) of Salmonella minnesota. Humans are regularly exposed to LPS produced by naturally existing bacteria because many

Table 5 Comparison of 4vHPV versus other HPV vaccines regarding solicited local and systemic symptoms

\begin{tabular}{lllll}
\hline Components & Studies, $\mathrm{n}$ & Subjects, $\mathrm{n}$ & Pooled RR $[95 \% \mathrm{Cl}]$ & Reference \\
\hline Pain & 3 & 1845 & $0.89[0.75,1.05]$ & {$[28,29,45]$} \\
Redness & 2 & 1246 & $0.70[0.46,1.07]$ & {$[28,29]$} \\
Swelling & 3 & 1845 & $0.71[0.57,0.88]$ & {$[28,29,45]$} \\
Fatigue & 2 & 1250 & $0.82[0.73,0.92]$ & {$[28,29]$} \\
\hline
\end{tabular}

Other HPV vaccines stand for $2 \mathrm{vHPV}$ and $9 \mathrm{vHPV}$

Two studies [28, 29] compare 4vHPV with 2vHPV and one study [45] compares $4 \mathrm{vHPV}$ with 9vHPV

Abbreviations: HPV human papillomavirus, 2vHPV bivalent human papillomavirus, $4 \mathrm{VHPV}$ quadrivalent human papillomavirus, $R R$ risk ratio, $\mathrm{Cl}$ confidence interval bacterial species contain LPS as a major component of the bacterial cell wall [50]. AS-04 is contained only in 2vHPV vaccine among marketed HPV vaccines in Japan. On the other hand, an AS-04-containing hepatitis B vaccine (Fendrix ${ }^{\circ}$, GlaxoSmithKline Biologicals) has shown noninferiority in safety (including solicited local symptoms and systemic symptoms) compared to a standard hepatitis $B$ vaccine that does not contain AS-04 [51]. Osmotic pressure and $\mathrm{pH}$ of HPV vaccines are similar to other subcutaneous vaccines (2vHPV: $\mathrm{pH}$ 6.0-7.0, osmotic pressure ratio 1.0; 4vHPV: $\mathrm{pH} 5.7-6.7$, osmotic pressure ratio 2.0 ). The difference in pharmaceutical property may not affect the safety profile in our meta-analysis.

Mixing aluminum salts in vaccines is a conventional method of non-specific proinflammatory augmentation of immune response and has been widely used as an adjuvant for vaccines [52]. According to a meta-analysis on adverse events after administration of aluminumcontaining vaccines to children aged 10 to 16 years, there is no significant association between exposure to aluminum-containing vaccines and local induration/ swelling and local pain lasting up to 14 days [53]. Furthermore, aluminum-containing vaccines other than HPV vaccines (including influenza, pneumococcal, and hepatitis $B$ vaccines) have been widely used in clinical practice, and the amount of aluminum in these vaccines ranged from 110 to $500 \mu \mathrm{g}$. All included studies except for one study [35] contained aluminum in control group, however, a sensitivity analysis showed there was no discrepancy after removal of the study. 
Racial difference may contribute to harmful responses to vaccines. A meta-analysis for safety of HPV vaccines in Asian population concluded that HPV vaccine (2vHPV or 4vHPV) had slightly higher risk of solicited local and systemic symptoms compared to controls (placebo or hepatitis vaccine) [16]. In our subgroup analysis, Asian population tends to have a slightly higher incidence of solicited local symptoms such as pain and swelling after $2 \mathrm{vHPV}$ vaccine compared to non-Asian population (pooled RR [95\% CI] for pain in Asians and non-Asians: 1.37 [1.30 to 1.45 ] and 1.24 [0.81 to 1.91 ], pooled RR [95\% CI] for swelling: $2.62[2.03,3.40]$ and 1.62 [1.33 to 1.98$]$, respectively, compared to the respective placebos). On the other hand, pooled RRs [95\% CI] for pain after $4 \mathrm{vHPV}$ vaccination were 1.23 [1.14 to 1.31] and 1.12 [1.06, 1.19] in Asian and nonAsian populations, respectively. To our knowledge, there is no study that investigates racial difference in incidence of solicited local and systemic symptoms. Further research is required to explain the racial difference.

There are some limitations in our study. First, our study did not focus on complex regional pain syndrome and chronic pain as pain-related symptoms after HPV vaccination. Second, we did not evaluate the efficacy of HPV vaccines systematically. However, many reports have shown the preventive efficacy of HPV vaccines [26-47]. The prevalence of HPV among women aged 14 to 19 years has decreased by $64 \%$ since vaccination was introduced a decade ago in the United States [54]. Third, adverse reactions after vaccination were selfreported in all included studies except for one report [44]. We performed a sensitivity analysis and confirmed that the removal of the report did not alter our results and conclusion.

\section{Conclusions}

Our meta-analysis revealed that HPV vaccines had significantly higher risk of any injection site symptom compared to placebo or other vaccines (hepatitis A and $B$ vaccines), and the incidence of solicited local symptoms was no difference between $2 \mathrm{vHPV}$ vaccination and $4 \mathrm{vHPV}$ vaccination. However, the most adverse reactions were transient.

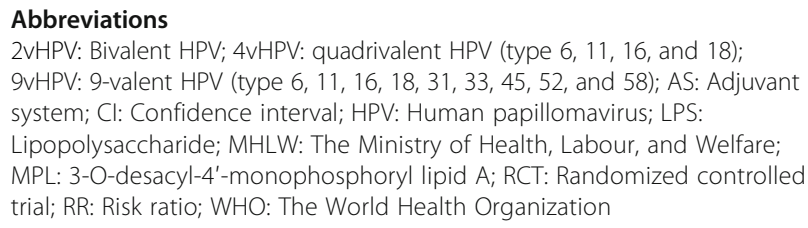

Acknowledgements

Not applicable.

Funding

All authors received no financial support for this study.

\section{Availability of data and materials}

The dataset used for this meta-analysis are available from the corresponding author on reasonable request.

\section{Authors' contributions}

All authors conceived and designed the study. YO and HT conducted the literature search and were responsible for statistical analysis of the data. All authors involved in the interpretation of data. $\mathrm{YO}, \mathrm{HT}$, and $\mathrm{RO}$ drafted the manuscript. RO and KM contributed to discussions. All authors read and approved the final manuscript.

Ethics approval and consent to participate

Not applicable.

\section{Consent for publication}

Not applicable.

\section{Competing interests}

The authors declare that they have no competing interests.

\section{Publisher's Note}

Springer Nature remains neutral with regard to jurisdictional claims in published maps and institutional affiliations.

\section{Author details}

${ }^{1}$ Faculty of Pharmacy, Department of Pharmaceutical Sciences, Musashino University, 1-1-20 Shinmachi Nishitokyo-shi, Tokyo 202-8585, Japan.

${ }^{2}$ Department of Pharmacotherapy, Meiji Pharmaceutical University, 2-522-1 Noshio, Kiyose-shi, Tokyo 204-8588, Japan.

Received: 15 March 2017 Accepted: 27 June 2017

Published online: 11 July 2017

References

1. Parkin DMBF, Ferlay J, Pisani P. Global cancer statistics, 2002. CA Cancer J Clin. 2005;55:74-108.

2. Kamangar FDG, Anderson WF. Patterns of cancer incidence, mortality, and prevalence five continets: defining priorities to reduce cancer disparities in different geographic regions of the world. J Clin Oncol. 2006;24:2137-50.

3. World Health Organization. International Agency for research on Cancer, Lyon. 2012. http://globocan.iarc.fr/Pages/fact_sheets_cancer.aspx. Accessed 12 Feb 2017.

4. Cancer Registry and Statistics. Cancer Information Service, National Cancer Center, Japan. 2015. http://ganjoho.jp/reg_stat/statistics/dl/index.html. Accessed 10 June 2017.

5. Sobue T, editor. White Paper Cancer Statistics 2012. Tokyo: Shinohara Press; 2012.

6. Kjaer SK, Frederiksen K, Munk C, Iftner T. Long-term absolute risk of cervical intraepithelial neoplasia grade 3 or worse following human papillomavirus infection: role of persistence. J Natl Cancer Inst. 2010;102(19):1478-88.

7. Rodriguez AC, Schiffman M, Herrero R, Hildesheim A, Bratti C, Sherman ME, et al. Longitudinal study of human papillomavirus persistence and cervical intraepithelial neoplasia grade 2/3: critical role of duration of infection. I Natl Cancer Inst. 2010;102(5):315-24.

8. Smith JS, Lindsay L, Hoots B, Keys J, Franceschi S, Winer R, et al. Human papillomavirus type distribution in invasive cervical cancer and high-grade cervical lesions: a meta-analysis update. Int J Cancer. 2007;121(3):621-32.

9. Bao YP, Li N, Smith JS, Qiao YL, ACCPAB Members. Human papillomavirus type distribution in women from Asia: a meta-analysis. Int J Gynecol Cancer. 2008;18(1):71-9.

10. Lehtinen M, Dillner J. Clinical trials of human papillomavirus vaccines and beyond. Nat Rev Clin Oncol. 2013;10(7):400-10.

11. World Health Organization. Human Papillomavirus vaccines WHO Position Paper. Wkly Epidemiol Rec. 2009:84(15):117-32.

12. Gilmour S, Kanda M, Kusumi E, Tanimoto T, Kami M, Shibuya K. HPV vaccination programme in Japan. Lancet. 2013;382(9894):768.

13. Morimoto A, Ueda Y, Egawa-Takata T, Yagi A, Terai Y, Ohmichi M, et al. Effect on HPV vaccination in Japan resulting from news report of adverse events and suspension of governmental recommendation for HPV vaccination. Int J Clin Oncol. 2015;20(3):549-55. 
14. Ueda Y, Enomoto T, Sekine M, Egawa-Takata T, Morimoto A, Kimura T. Japan's failure to vaccinate girls against human papillomavirus. Am J Obstet Gynecol. 2015;212(3):405-6.

15. Lu B, Kumar A, Castellsague X, Giuliano AR. Efficacy and safety of prophylactic vaccines against cervical HPV infection and diseases among women: a systematic review \& meta-analysis. BMC Infect Dis. 2011;11:13.

16. Setiawan DLJ, Pouwels KB, Wilschut JC, Postma MJ. Immunogenicity and safety of human papillomavirus (HPV) vaccination in Asian populations from six countries: a meta-analysis. Jpn J Clin Oncol. 2017;47(3):265-76.

17. Pharmaceuticals and Medical Devices Agency. Tokyo. https://www.pmda.go. jp/index.html. Accessed 12 Feb 2017.

18. Cunningham AL, Lal H, Kovac M, Chlibek R, Hwang SJ, Diez-Domingo J, et al. Efficacy of the herpes zoster subunit vaccine in adults 70 years of age or older. N Engl J Med. 2016;375(11):1019-32.

19. Demeulemeester M, Lavis N, Balthazar Y, Lechien P, Heijmans S. Rapid safety assessment of a seasonal intradermal trivalent influenza vaccine. Hum Vaccin Immunother. 2017;13(4):889-94.

20. Embree J, Law B, Voloshen T, Tomovici A. Immunogenicity, safety, and antibody persistence at 3, 5, and 10 years postvaccination in adolescents randomized to booster immunization with a combined tetanus, diphtheria, 5-component acellular pertussis, and inactivated poliomyelitis vaccine administered with a hepatitis B virus vaccine concurrently or 1 month apart. Clin Vaccine Immunol. 2015;22(3):282-90.

21. Huu TNTN, Tuan HM, Viet HL, Le Thanh BP, Yu TW, Shafi F, Habib A, Borys D. Safety and reactogenicity of primary vaccination with the 10-valent pneumococcal nontypeable Haemophilus influenzae protein D conjugate vaccine in Vietnamese infants: a randomised, controlled trial. BMC Infect Dis. 2013:13(95).

22. Leroux-Roels GHE, Maes C, Levy J, De Boever F, Licini L, David MP, Dobbelaere K, Descamps D. Randomized trial of the immunogenicity and safety of the Hepatitis B vaccine given in an accelerated schedule coadministered with the human papillomavirus type 16/18 AS04-adjuvanted cervical cancer vaccine. Clin Vaccine Immunol. 2011:18(9):1510-8.

23. Leroux-Roels GMC, De Boever F, Traskine M, Rüggeberg JU, Borys D. Safety, reactogenicity and immunogenicity of a novel pneumococcal protein-based vaccine in adults: a phase 1/II randomized clinical study. Vaccine. 2014;32:6838-46.

24. Higgins JPTGS. In: JPT AD H, editor. Cochrane handbook for systematic reviews of interventions. Chichester: Willy; 2008. p. 194-206.

25. Higgins JPTS, Deeks JJ, Altman DG. Measuring inconsistency in meta-analyses. BMJ. 2003;327:557-60.

26. Gardasil approval documentation. In: pharmaceuticals and Medical Devices Agency. 2011. http://www.pmda.go.jp/drugs/2011/P201100122/index.html. Accessed 12 Feb 2017

27. Bhatla N, Suri V, Basu P, Shastri S, Datta SK, Bi D, et al. Immunogenicity and safety of human papillomavirus-16/18 AS04-adjuvanted cervical cancer vaccine in healthy Indian women. J Obstet Gynaecol Res. 2010;36(1):123-32.

28. Draper E, Bissett SL, Howell-Jones R, Waight P, Soldan K, Jit M, et al. A randomized, observer-blinded immunogenicity trial of Cervarix((R)) and Gardasil((R)) Human Papillomavirus vaccines in 12-15 year old girls. PLoS One. 2013;8(5):e61825.

29. Einstein MH, Baron M, Levin MJ, Chatterjee A, Edwards RP, Zepp F, et al. Comparison of the immunogenicity and safety of Cervarix ${ }^{\mathrm{TM}}$ and Gardasil ${ }^{\oplus}$ human papillomavirus (HPV) cervical cancer vaccines in healthy women aged 18-45 years. Human Vaccines. 2009;5(10):705-19.

30. Group FIS. Quadrivalent vaccine against human papillomavirus to prevent high-grade cervical lesions. N Engl J Med. 2007;356:1915-27.

31. Harper DMFE, Wheeler C, Ferris DG, Jenkins D, Schuind A, Zahaf $T$, Innis B, Naud P, De Carvalho NS, Roteli-Martins CM, Teixeira J, Blatter MM, Korn AP, Quint W, Dubin G, GlaxoSmithKline HPV Vaccine Study Group. Efficay of a bivalent L1 virus-like particle vaccine in prevention of infection wiht human papillomavirus types 16 and 18 in young woumen: a randomised controlled trial. Lancet. 2004;364:1757-65.

32. Kang S, Kim KH, Kim YT, Kim YT, Kim JH, Song YS, et al. Safety and immunogenicity of a vaccine targeting human papillomavirus types 6, 11 16 and 18: a randomized, placebo-controlled trial in 176 Korean subjects. Int J Gynecol Cancer. 2008;18(5):1013-9.

33. Kim SC, Song YS, Kim YT, Kim YT, Ryu KS, Gunapalaiah B, et al. Human papillomavirus 16/18 AS04-adjuvanted cervical cancer vaccine: immunogenicity and safety in 15-25 years old healthy Korean women. J Gynecol Oncol. 2011;22(2):67-75.

34. Kim YJ, Kim KT, Kim JH, Cha SD, Kim JW, Bae DS, et al. Vaccination with a human papillomavirus (HPV)-16/18 AS04-adjuvanted cervical cancer vaccine in Korean girls aged 10-14 years. J Korean Med Sci. 2010;25(8):1197-204.
35. Konno RDK, Godeaux OO, Tamura S, Yoshikawa H. Immunogenicity, reactogenicity, and safety of human papillomavirus 16/18 AS04-adujuvanted vaccine in Japanese Women. Int J Gynecol Cancer. 2009;19:905-11.

36. Lim Bk NK, Omar J, Omar SZ, Gunapalaiah B, Teoh YL, Bock HL, Bi D. Immunogenicity and safety of the AS04-adjuvanted human papillomavirus16/18 cervical cancer vaccine in Malaysian women aged 18-35 years: a randomized controlled trial. Med J Malaysia. 2014;69(1):2-8.

37. Medina DM, Valencia A, de Velasquez A, Huang LM, Prymula R, Garcia-Sicilia J, et al. Safety and immunogenicity of the HPV-16/18 AS04-adjuvanted vaccine: a randomized, controlled trial in adolescent girls. J Adolesc Health. 2010;46(5):414-21.

38. Mugo N, Ansah NA, Marino D, Saah A, Garner El. Evaluation of safety and immunogenicity of a quadrivalent human papillomavirus vaccine in healthy females between 9 and 26 years of age in Sub-Saharan Africa. Hum Vaccin Immunother. 2015;11(6):1323-30.

39. Muñoz NMRJ, Pitisuttithum P, Tresukosol D, Monsonego J, Ault K, Clavel C, Luna J, Myers E, Hood S, Bautista O, Bryan J, Taddeo FJ, Esser MT, Vuocolo S, Haupt RM, Barr E, Saah A. Safety, immunogenicity, and efficacy of quadrivalent human papillomavirus $($ type6,11,16,18) recombinat vaccine in women aged 24-45 years: a randomised, double-blind trial. Lancet. 2009;373:1949-57.

40. Ngan HYSCA, Tam KF, Chan KKL, Tang HW, Bi D. Human papillomavirus-16/ 18 AS04-adjuvanted cervical cancer vaccine: immunnogenicity and safety in healthy Chinese women from Hong Kong. Hong Kong Med J. 2010;16(3):171-9.

41. Paavonen J, Jenkins D, Bosch FX, Naud P, Salmerón J, Wheeler CM, et al. Efficacy of a prophylactic adjuvanted bivalent L1 virus-like-particle vaccine against infection with human papillomavirus types 16 and 18 in young women: an interim analysis of a phase III double-blind, randomised controlled trial. Lancet. 2007;369(9580):2161-70.

42. Perez G, Lazcano-Ponce E, Hernandez-Avila M, Garcia PJ, Munoz N, Villa LL, et al. Safety, immunogenicity, and efficacy of quadrivalent human papillomavirus (types $6,11,16,18)$ L1 virus-like-particle vaccine in Latin American women. Int J Cancer. 2008;122(6):1311-8.

43. Skinner SR, Szarewski A, Romanowski B, Garland SM, Lazcano-Ponce E, Salmerón J, et al. Efficacy, safety, and immunogenicity of the human papillomavirus 16/18 AS04-adjuvanted vaccine in women older than 25 years: 4-year interim follow-up of the phase 3, double-blind, randomised controlled VIVIANE study. Lancet. 2014;384(9961):2213-27.

44. Sow PS, Watson-Jones D, Kiviat N, Changalucha J, Mbaye KD, Brown J, et al. Safety and immunogenicity of human papillomavirus-16/18 AS04adjuvanted vaccine: a randomized trial in 10-25-year-old HIV-seronegative African girls and young women. J Infect Dis. 2013;207(11):1753-63.

45. Vesikari T, Brodszki N, van Damme P, Diez-Domingo J, Icardi G, Petersen LK, et al. A randomized, double-blind, phase III study of the immunogenicity and safety of a 9-valent human papillomavirus L1 virus-like particle vaccine (V503) versus gardasil(R) in 9-15-year-old girls. Pediatr Infect Dis J. 2015:34(9):992-8.

46. Villa LL, Ault KA, Giuliano AR, Costa RL, Petta CA, Andrade RP, et al. Immunologic responses following administration of a vaccine targeting human papillomavirus Types 6, 11, 16, and 18. Vaccine. 2006;24(27-28):5571-83.

47. Zhu F, Li J, Hu Y, Zhang $X$, Yang $X$, Zhao H, et al. Immunogenicity and safety of the HPV-16/18 AS04-adjuvanted vaccine in healthy Chinese girls and women aged 9 to 45 years. Hum Vaccin Immunother. 2014;10(7):1795-806.

48. Didierlaurent AM, Morel S, Lockman L, Giannini SL, Bisteau M, Carlsen H, et al. AS04, an aluminum salt- and TLR4 agonist-based adjuvant system, induces a transient localized innate immune response leading to enhanced adaptive immunity. J Immunol. 2009;183(10):6186-97.

49. Garçon N, Chomez P, Van Mechelen M. GlaxoSmithKline adjuvant systems in vaccines: concepts, achievements and perspectives. Expert Review of Vaccines. 2007:6(5):723-39.

50. Beutler BRE. Innate immune sensing and its roots: the story of endotoxin. Nat Rev Immunol. 2003:3(2):169-76.

51. Kong NC, Beran J, Kee SA, Miguel JL, Sanchez C, Bayas JM, et al. A new adjuvant improves the immune response to hepatitis $B$ vaccine in hemodialysis patients. Kidney Int. 2008;73(7):856-62.

52. Brewer JM. (How) do aluminium adjuvants work? Immunol Lett. 2006:102(1):10-5

53. Jefferson TRM, Di Pietrantonj C. Adverse events after immunisation with aluminium-containing DTP vaccines: systematic review of the evidence. Lancet Infect Dis. 2004;4(2):84-90.

54. Markowitz L LG, Hariri S, Steinau M, Dunne EF, Unger ER. Prevalence of HPV After Introduction of the vaccination program in the United States. Pediatrics 2016:137(3):e20151968 doi:10.1542/peds.2015-1968. Epub 2016 Feb 22. 